\title{
Unsupervised Classification of Mobile Device Images
}

\author{
Jocelin Rosales Corripio, Ana Lucila Sandoval Orozco, Luis Javier García Villalba \\ Group of Analysis, Security and Systems (GASS) \\ Department of Software Engineering and Artificial Intelligence (DISIA) \\ Faculty of Information Technology and Computer Science, Office 431 \\ Universidad Complutense de Madrid (UCM) \\ Calle Profesor José García Santesmases, 9 \\ Ciudad Universitaria, 28040 Madrid, Spain \\ Email: jocelinr@ucm.es, \{asandoval,javiergv\}@fdi.ucm.es
}

\begin{abstract}
As mobile devices are seeing widespread usage in the everyday life, the images from mobile devices can be used as evidence in legal purposes. Accordingly, the identification of mobile devices images are of significant interest in digital forensics. In this paper, we propose a method to determine the mobile devices camera source based on the grouping or clustering of images according to their source acquisition. Our clustering technique does not involve a priori knowledge of the number of images or devices to be identified or training data for a future classification stage. The proposal combines of hierarchical and flat clustering and the use of sensor pattern noise. Experimental results show that our approach is very promising for identifying mobile devices source.
\end{abstract}

\section{Keywords-Image Clustering; Image Forensics Analysis; PRNU; Sensor Pattern Noise}

\section{INTRODUCTION}

Nowadays, even suffering the impact of global financial crisis, the sales of mobile devices such as cell phones, smartphones or tablets, is still increasing. About $78.1 \%$ of mobile phones sold in 2010 have an integrated camera [1]. Integrated cameras in mobile devices outnumber traditional Digital Still Camera (DSCs). The sales of cameras integrated into mobile devices in 2013 exceeded 1800 million units. Similarly, there are predictions that the DSCs will disappear in favour of integrated mobile devices [2], since the quality of these cameras is growing at an unstoppable rate. Also, the emergence of cameras in mobile devices should not only be measured in sales figures, as in our daily life it is common to see how people use photographs from these devices for a variety of situations - personal life, news, legal evidence, software applications and so on. Therefore, forensic analysis of such images is particularly important in criminal investigations.

The image source acquisition identification and malicious tampering detection are of significant interest in digital image forensic analysis. This work focuses on the first branch. Also, since mobile device cameras have some characteristics that make them different from the rest, this work focuses on images from this type of devices. The source acquisition identification has closed scenarios and open scenarios approaches regarding. A closed scenario is one in which the image source identification is performed on a specific and known beforehand set of cameras. In closed scenario approach normally use to train and predict process in order to classify like Support Vector Machine (SVM) classifier. Instead, in open scenarios the forensic analyst does not know a priori the camera set to which images whose source identification will be identified belong.

In this paper, we propose a method that utilizes the hierarchical and flat clustering to image source identification in open scenarios. The objective of this approach is to group the different images into disjoint sets in which all their images belong to the same device. This approach is very close to reallife situations, since in many cases the set of cameras to which a set of images may belong is completely unknown to the analyst. In addition, it is virtually impossible to have a set of images to train a classifier with all mobile device cameras existing in the world. In this case, being able to group images into sets that belong to the same device is very useful, as this can provide very valuable and in some cases conclusive information to judicial investigators. The remainder of this paper is organized as follows. Section 2 briefly presents previous work related to forensic techniques for mobile device image source acquisition identification. The proposed technique is presented in section 3. The experiments and their results are presented in section 4 . Finally, in section 5 the conclusions drawn from this work are presented.

\section{A. Image Formation in Digital Cameras}

The first step is to understand and create image processing forensic algorithms is to thoroughly know the process of image acquisition in digital cameras. Fig. 1 summarizes this process. 


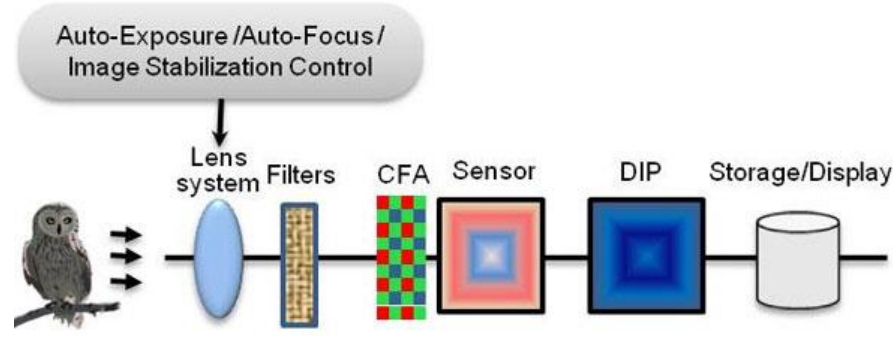

Fig. 1. Image acquisition process in digital cameras [3]

First, the lens system captures light from the scene by controlling the exposure, focus, and image stabilization. Next, the light passes through a set of filters that improve the visual quality of the image, and then the light gets to the image sensor called Color Filter Array (CFA); this is an array of light sensitive elements called pixels. Note that the choice of the CFA can influence the sharpness and the final appearance of the image since there are different CFA patterns.

The most commonly used model is the Green-Red-GreenBlue (GRGB) Bayer pattern; other models are: Red-GreenBlue-Emerald (RGBE), Cyan-Yellow-Yellow-Magenta (CYYM), Cyan-Yellow-Green-Magenta (CYGM) or RedGreen-Blue-White (RGBW). The incident light on the colored filters gets to a sensor which is responsible for generating an analogue signal proportional to the intensity of received light, keeping these values in an internal array.

There are currently two types of sensor technologies that meet this latter purpose in digital cameras: CCD (Charge Coupled Device) and CMOS (Complementary Metal Oxide Semiconductor). Both types of sensors essentially consist of Metal Oxide Semiconductors (MOS) and they work in a similar way, although the key difference is in the way in which pixels are scanned and the way in which the reading of the charges is carried out. CCD sensors need an additional chip to process the sensor's output information; this causes the manufacture of devices to be more costly and the sensors to be bigger. In contrast, CMOS sensors have independent active pixels, as they themselves perform the digitalization, offering speed and reducing the size and cost of the systems that make up a digital camera. Another difference between these two types of sensors is that the pixels in a CCD array capture light simultaneously, which promotes a more uniform output. CMOS sensors generally perform the reading as progressive scan (avoiding the blooming effect). CCD sensors are far superior to the CMOS in terms of noise and dynamic range; on the other hand, CMOS sensors are more sensitive to light and behave better in low light conditions. Early CMOS sensors were somewhat worse than CCDs, but nowadays this has been practically corrected.

The CCD technology has reached its limit and it is now when CMOS is being developed and its weaknesses are being overcome, so much that the majority of smartphones contain CMOS sensors. Signals stored by the CCD/CMOS sensor are then converted into a digital signal and transmitted to the image processor, once the image processor receives the digital signal it eliminates noise and other introduced anomalies. Some other processes applied to the signal are color interpolation, gamma correction, and color correction.

\section{PREVIOUS WORKS IN IMAGE FORENSIC ANALYSIS}

Most research on image source acquisition identification focuses on traditional digital cameras or DSC; most of these techniques are not valid for mobile device images. In [4] an overview of this research can be seen.

For any type of image classification, either in open or closed scenarios, it is necessary to obtain certain features that allow classification techniques to perform their task. According to [3], four groups of techniques can be established for this purpose: based on lens aberration, based on the CFA matrix interpolation, based on the sensor imperfections and based on the use of image features. Within the latter group a subdivision can be made based on color features, quality features, and wavelet domain statistics. This work uses techniques based on sensor imperfections, particularly those based on the sensor pattern noise (SPN). The main components of image noise are the Fixed Pattern Noise (FPN) and the Photo Response Non Uniformity (PRNU). There are several sources of imperfections and noise introduced at different stages of the creating pipeline of an image in a digital camera. Even if a uniform and fully lighted picture is taken it is possible to see small changes in the intensity between pixels. This is due to the shot noise is random and, in large part, the pattern noise is deterministic and is kept approximately equal if several pictures of the same scene are taken.

The analysis of clusters, or clustering, aims to group a collection of objects into representative classes called clusters, without a priori information, in such a way that the objects belonging to each cluster keep a greater similarity to objects from other clusters. Image grouping can be performed using supervised or unsupervised learning techniques. In the first case it is essential to know the device information a priori, i.e., it is clearly identified with the classification in closed scenarios which requires a training stage with the features extracted from the images and a second classification stage in accordance with the previous result. However, in a real case it may be difficult to have the camera in question or a set of photographs taken by it to carry out training, hence the need for unsupervised learning techniques, which directly correspond to open scenarios.

Traditional clustering has been known to be an unsupervised learning technique; however, there are some cases of supervised clustering where it is possible to apply an anterior or posterior approach to improve the grouping itself. This is to prevent that elements of different classes are in the same cluster, which requires having a priori knowledge of the data set. This issue is addressed in [6], although it is worth mentioning that this article is focused on the use of unsupervised techniques.

In order to determine the similarity between objects belonging to the same cluster, there are distance measures such as Euclidean distance, Manhattan distance, and Chebychev distance, among others. Alternatively, it is possible to use 
similarity functions $S\left(X_{i}, X_{j}\right)$ which compare two vectors $X_{i}$ and $X_{j}$ symmetrically, i.e., $S\left(X_{i}, X_{j}\right)=S\left(X_{j}, X_{i}\right)$. These functions reach their highest values as $X_{i}$ and $X_{j}$ are more similar. One of the most commonly used measures in image source identification is normalized correlation [7][8][17] defined in equation 1.

$$
\operatorname{corr}\left(X_{i}, X_{j}\right)=\frac{\left(X_{i}-\overline{X_{i}}\right) \odot\left(X_{j}-\overline{X_{j}}\right)}{\left\|X_{i}-\overline{X_{i}}\right\| \cdot\left\|X_{j}-\overline{X_{j}}\right\|}
$$

Where $\overline{X_{i}}$ and $\overline{X_{j}}$ represent the mean vector, $X_{i} \odot \overline{X_{j}}$ is the scalar product of two vectors and $\left\|X_{i}\right\|$ is the $L_{2}$ norm of $X_{i}$.

According to the clustering algorithms classification proposed in [9], we find the hierarchical methods whose purpose is to achieve a structure called dendogram which represents the grouping of objects according to their levels of similarity. This grouping can be done in different ways: agglomerative or decisive. Agglomerative grouping initially considers each object as a separate class until iteratively grouping all the objects in a single class. Divisive clustering is based on the idea of starting from a single class until managing to separate all objects into individual classes. There are also partitioning algorithms, wherein starting a partition, the algorithm takes care of moving objects from one cluster to another to minimize certain error criterion. Within this category, the most famous method is k-means; however, most of these methods require knowing in advance the number of clusters, which is why they are not widely used in forensic image analysis. Finally, there are other clustering algorithms such as: [10] which produces clusters by means of graphs, [11] based on the density where the points within a cluster are given by a certain probability function, clusters based on models such as decision trees [12] or neural networks [13] and clustering with soft-computing methods such as fuzzy clustering [14], evolutionary clustering methods and simulated annealing clustering [15].

There are previous works on image grouping by unsupervised methods; all of them consider SPN as the most reliable criterion for representing a device's digital footprint, hence the PRNU is used specifically as a footprint and normalized correlation as a similarity measure to achieve image grouping by device.

[16] uses a classification technique with unsupervised learning where grouping is achieved by graph maximization. Clustering is performed from not-oriented graph with weights, starting with an affinity matrix where the connection weights between vertices is the correlation value between each SPN, starting with a random node. In each iteration, the remaining nodes are connected and the nodes closest to the central one are chosen, obtaining a new affinity matrix in each step; the algorithm stops when the number of closest nodes is less than a $\$ \mathrm{k} \$$ parameter. Subsequently, the graph is partitioned to the point where similarity in a set is maximum and minimum with respect to other sets.

In [8] clusters are performed using Markov random fields. A clustering algorithm based on matrix containing all the correlations between the SPN of several cameras is proposed.
In each iteration the algorithm groups within classes the most similar SPNs making use of the local features of Markov random fields and assigns a new class label to each SPN maximizing a probability function, the criterion to stop the algorithm is satisfied when there are no label changes after a certain number of iterations.

The algorithm proposed in [17] and on which this research is based uses hierarchical clustering to group images. Prior to the clustering algorithm, the authors apply a function for sensor noise improvement, which strengthens the lower components and attenuates the high components in the wavelet domain in order to remove the scene details in it. With a similarity matrix containing all the correlations between different SPNs and taking as a starting point each image as a single cluster, the clustering algorithm groups the two clusters with the highest correlation value forming a single cluster and updates the matrix with a new row and column that replace the rows and columns of the grouped clusters. The link criterion chosen to mix two clusters was average linkage. In each iteration of the algorithm, cluster status at that time is stored on a partition and the global silhouette coefficient is calculated. At the end of the algorithm the partition whose silhouette coefficient value is the lowest is chosen, the number of clusters at that point should correspond to the number of devices that exist initially, as well as the content of each cluster to the SPN for each device. The authors carry out a training stage with the described algorithm and a classification stage for the remaining images, for this it is sufficient to obtain the average of the SPNs for each cluster and compare them against the remaining images, the image will be classified within the cluster whose correlation is highest.

\section{TECHNIQUE DESCRIPTION}

The proposed unsupervised clustering algorithm is based on the one proposed in [17]. It is a combination of a hierarchical clustering, and a flat clustering. That is, despite forming a dendrogram structure with each iteration of the algorithm, at the end the clusters are taken as unrelated entities since each of them must correspond to a specific device.

Prior to performing the clustering, it is necessary to obtain sensor pattern noises of the image set $I$ using the extraction algorithm and the parameter of noise suppression $s_{0}=5$ proposed in [5]. Equation 2 shows this calculation.

$$
\mathrm{n}^{(\mathrm{i})}=\mathrm{I}^{(\mathrm{i})}-\mathrm{F}\left(\mathrm{I}^{(\mathrm{i})}\right)
$$

Where $i=1, \ldots, N, N$ is the number of images, $n^{(i)}$ is the noise pattern of each image $i, I^{(i)}$ is the image with sensor noise of each image $i$ and $F$ is the noise removal filter based on wavelet transform. For this, the algorithm developed by Goljan et al. in [18] was used. No noise improvement algorithm, such as those proposed by [8] and [17], has been used in our proposal. The Wiener filter in the frequency domain is sufficient to remove most of the scene details that are present when extracting the SPN.

For each of the $N$ noises $n_{1}, \ldots, n_{N}$ the correlation value is obtained using equation 1 and this generates a similarity matrix $H$ of $N \times N$. This matrix is symmetric and consists of ones in 
its main diagonal (since the correlation of noise with itself is 1). Once the matrix has been generated it will not be necessary to recalculate the correlations between noises along the clustering algorithm, saving time and processing power.

The selected hierarchical clustering algorithm involves finding within the $H$ matrix the noise pair $k$ and $l$ with a highest correlation value. It is worth mentioning that the correlation values in the main diagonal are not taken into account. Then the rows and columns $k$ and $l$ are deleted and both a new row and a new column are added to the matrix. These new row and column values are the result of a linkage criterion. The function chosen for this work was the average linkage method since its results are more satisfactory than with other linkage methods such as single linkage or complete linkage, as is suggested in [17]. Equation 3 shows the function of the average linkage method between two clusters A and B.

$$
\mathrm{H}(\mathrm{A}, \mathrm{B})=\frac{1}{\|\mathrm{~A}\|\|\mathrm{B}\|} \sum_{\mathrm{n}_{\mathrm{i}} \in \mathrm{A}, \mathrm{n}_{\mathrm{j}} \in \mathrm{A}} \operatorname{corr}\left(\mathrm{n}_{\mathrm{i}}, \mathrm{n}_{\mathrm{j}}\right)
$$

where the $\operatorname{corr}\left(n_{i}, n_{j}\right)$ value is calculated with equation 1 and can be taken from the matrix $H$ to simplify the computational processing. $\|A\|$ and $\|B\|$ is the cardinality of the $A$ and $B$ clusters respectively.

Each iteration of the algorithm takes the two clusters with the highest correlation value in the matrix and mixes the objects contained in them to create a new cluster, while storing the state of the different clusters in partition $P_{0}, \ldots, P_{N-1}$ with the aim of knowing the contents of the cluster at any time. In the hierarchical clustering, the final result of the algorithm is a cluster containing all objects. However, in this work each cluster should represent a device at the end of the execution. For this reason, the silhouette coefficient as a measure of validation of clusters was used. The silhouette coefficient measures the similarity index between the elements of a single cluster (cohesion) and the similarity between the elements of a cluster with respect to the others (separation). Unlike Caldelli et al. [17], in our proposal the calculation of the silhouette coefficient is performed for each cluster contained in the $P_{i}$ partition and not for each pattern noise, as noted in Equation 4.

$$
s_{j}=\max \left(b_{j}\right)-a_{j}
$$

where $a_{j}$ (cohesion) is the average correlation between all noise patterns within the $c_{j}$ cluster. $b_{j}$ (separation) is the average correlation of noise patterns contained in the $c_{j}$ cluster with respect to noise patterns in the remaining clusters. The nearest neighboring cluster is taken, namely the one with the highest correlation.

For each iteration $q$ of the algorithm a global measure of all the silhouette coefficients calculated from the $K$ clusters is obtained, this is equivalent to averaging the $s_{j}$ values in $q$. Equation 5 shows this calculation.

$$
\mathrm{SC}_{\mathrm{q}}=\frac{1}{\mathrm{~K}} \sum_{\mathrm{j}=1}^{\mathrm{K}} \mathrm{S}_{\mathrm{j}}
$$

Upon completion of the hierarchical clustering, the $S C_{q}$ with the lowest value is searched for, which indicates that the partition $P_{q}^{*}$ clusters are at a greater correlation level. The number of clusters at that moment should correspond to the actual number of devices. The aim of storing the partition at each time of the algorithm is to avoid rerunning the clustering because information of all the clusters in each iteration $q$ is known. Next algorithm shows the proposal's pseudocode.

1. Calculate $n^{(i)}$ of each image where $i \in 1, \ldots, N$;

2. Generate the similarity matrix $H \in R^{N \times N}$;

3. Foreach $q \in 1, \ldots, N-1$ do

4. Find cluster $H(k, l)$ with the highest similarity;

5. Remove the pair of rows and columns corresponding to clusters $k$ and $l$;

6. Calculate the values of the new cluster using average link criteria and add the row and its corresponding column;

7. Determine the overall silhouette coefficient $S C_{q}$;

8. Store the partition $P_{q}$;

9. Find the partition where $\min _{q}\left(S C_{q}\right)$.

\section{EXPERIMENTS AND RESULTS}

The experiments were performed with a total set of 1050 photographs from 7 different mobile device camera models. The total set contains 150 photographs from each model. 7 devices are from different manufacturers (Apple iPhone 5, Huawei U8815, LG E400, Samsung GTS5830M, Zopo ZP980, Sony ST25a and Nokia 800 Lumia).

All the images were cropped to $1024 \times 1024$ pixels, all images have a horizontal orientation. The scenes of the photographs were chosen randomly, both indoors and outdoors, and they were also taken at different times and places in order to simulate a more realistic scenario. In the extraction of the noise pattern from all images, the zero - mean of rows and columns was used, 3 RGB color channels were converted to a single matrix in grayscale. Additionally, all experiments were conducted using the Wiener filter in the frequency domain.

To measure the degree of certainty in the results, the true positive rate TPR was used. The mean TPR for each of the following experiments is calculated, computing for each cluster the number of photos that have been well classified (TPR of each cluster) and averaging the TPRs of all the resulting clusters (if there are fewer clusters than devices the average takes into account the number of devices). To calculate the TPR of each cluster, the device that has the largest number of images with respect to the total of images by device needs to be identified within the cluster, that being the predominant device cluster, then calculate the percentage of photos that have been well classified for that device in the cluster. Actually, in the vast majority of cases it can be seen that a cluster is associated with one or more devices, as it can observed in matrices such as the ones in Tables I, II and III. If there are multiple clusters with the same number of photos from a device or a cluster with the same number of photos from several devices and in turn these being the highest, the cluster that is taken as predominant for the device is one chosen among the different options. It may be the case that if there is an extra cluster, a cluster may 
not be predominant for any device (see Table II) and its TPR for this cluster is 0 . Or there might be one less cluster (see Table III), in this case the association of the cluster to a device will be taken into account and the number of devices will be used to calculate the average, as described above.

In Tables I, II and III there are examples that illustrate the calculation of the TPR for the three cases that may occur.

TABLE I. TPR WITH EQUAL NUMBER OF DEVICES THAN CLUSTERS

\begin{tabular}{|c|c|c|c|c|c|c|}
\hline \multirow{2}{*}{ Brand - Model } & \multicolumn{5}{|c|}{ Clusters (\%) } & \multirow{2}{*}{$\begin{array}{c}\text { Average } \\
\text { TPR }\end{array}$} \\
\cline { 2 - 6 } & $\mathbf{1}$ & $\mathbf{2}$ & $\mathbf{3}$ & $\mathbf{4}$ & $\mathbf{5}$ & \\
\hline Apple Iphone 5 & 49 & 0 & 0 & 1 & 0 & \\
\hline Huawei U8815 & 0 & 50 & 0 & 0 & 0 & \\
\hline LG E400 & 0 & 1 & 49 & 0 & 0 & \\
\hline Nokia 800 Lumia & 0 & 0 & 0 & 50 & 0 & \\
\hline Samsung GT5830m & 0 & 0 & 0 & 0 & 50 & \multirow{2}{*}{$\mathbf{9 9 . 2 \%}$} \\
\hline TPR by cluster & $\mathbf{9 8}$ & $\mathbf{1 0 0}$ & $\mathbf{9 8}$ & $\mathbf{1 0 0}$ & $\mathbf{1 0 0}$ & \\
\hline
\end{tabular}

In the results of the experiments 3 possible cases are considered: a) The number of identified clusters is equal to the number of devices, b) the number of identified clusters is higher than the number of devices, and c) the number of identified clusters is lower than the number of devices. Although the first case is ideal, in the second case classifications that do not mix different types of devices in a same cluster can be obtained.

TABLE II. TPR WITH LESS NUMBER OF DEVICES THAN CLUSTERS

\begin{tabular}{|c|c|c|c|c|c|}
\hline \multirow{2}{*}{ Brand - Model } & \multicolumn{4}{|c|}{ Clusters } & \multirow{2}{*}{$\begin{array}{c}\text { Average } \\
\text { TPR }\end{array}$} \\
\hline & 1 & 2 & 3 & 4 & \\
\hline Apple I-phone 5 & 100 & 0 & 0 & 0 & \\
\hline Huawei -U8815 & 0 & 100 & 0 & 0 & \\
\hline LG -E400 & 0 & 0 & 97 & 3 & \\
\hline TPR by cluster & 100 & 100 & 97 & $\mathbf{0}$ & $99 \%$ \\
\hline
\end{tabular}

TABLE III. TPR WITH MORE NUMBER OF DEVICES THAN CLUSTERS

\begin{tabular}{|c|c|c|c|c|c|}
\hline \multirow{2}{*}{ Brand - Model } & \multicolumn{4}{|c|}{ Clusters (\%) } & \multirow{2}{*}{$\begin{array}{c}\text { Average } \\
\text { TPR }\end{array}$} \\
\hline & 1 & 2 & 3 & 4 & \\
\hline Apple Iphone 5 & 100 & 0 & 0 & 0 & \\
\hline Huawei U8815 & 0 & 100 & 0 & 0 & \\
\hline LG E400 & 0 & 0 & 100 & 0 & \\
\hline Nokia 800 Lumia & 100 & 0 & 0 & 0 & \\
\hline Samsung GT 5830M & 0 & 0 & 0 & 100 & \\
\hline TPR by cluster & 100 & 100 & 100 & 100 & $80 \%$ \\
\hline
\end{tabular}

This paper has made an analysis of the main unsupervised image grouping techniques, which are of utmost importance in digital image forensic analysis. Despite the rise of mobile device cameras these days, there are still few references for unsupervised mobile device image grouping in the state of the art. Most of the works refer to the supervised classification and in many cases they are not focused on mobile device images, which have unique characteristics. The noise added in every photograph by the camera sensor, due to the faults in its manufacturing process or defects from daily use, has proven to be a reliable source of device identification. Likewise, the calculation of normalized correlation between sensor noises extracted from two or more pictures is also a measure of similarity commonly used in unsupervised image learning techniques, clustering techniques being the ones which obtain the best results. The algorithm of this proposal is based on the combination of a hierarchical clustering and a flat clustering for the separation between clusters. The use of the silhouette coefficient for cluster validation proved to report good results when obtaining high TPRs; also, the number of clusters corresponded to the number of actual devices in most cases. Experiments conducted in this work have revealed a great diversity of situations with regard to the symmetry or not of the photo sets, their size, the number of devices used and the use of devices of the same brand. After all the experiments, it is concluded that the results of the application of the technique are good $(92.7 \%$ TPR on average for all the experiments).

\section{ACKNOWLEDGMENT}

The research leading to these results has been partially funded by the European Union's H2020 Program under the project SELFNET (671672). Part of the computations of this work was performed in EOLO, the HPC of Climate Change of the International Campus of Excellence of Moncloa, funded by MECD and MICINN. This work was supported by the "Programa de Financiación de Grupos de Investigación UCM validados de la Universidad Complutense de Madrid - Banco Santander".

\section{REFERENCES}

[1] J. Hsu, "The Worldwide Mobile Phone Camera Module Market and Taiwan's Industry, 2010 and Beyond", 2010, pp. 1-18.

[2] R. Baer, "Resolution Limits in Digital Photography: The Looming End of the Pixel Wars - OSA Technical Digest (CD)", in Proceedings of the Imaging Systems, Tucson, Arizona United States, June 2010.

[3] T. Van Lanh, K.S. Chong, S. Emmanuel, M.S. Kankanhalli, "A Survey on Digital Camera Image Forensic Methods", in Proceedings of the IEEE International Conference on Multimedia and Expo, Beijing, pp. 16-19, July 2007.

[4] A. L. Sandoval Orozco, D. M. Arenas González, J. Rosales Corripio, L. J. García Villalba, J. C. Hernandez-Castro, "Techniques for Source Camera Identication", in Proceedings of the 6th International Conference on Information Technology, pp. 1-9, May 2013.

[5] J. Lukas, J. Fridrich, M. Goljan, "Digital Camera Identification from Sensor Pattern Noise", IEEE Transactions on Information Forensics and Security, IEEE, 2006, vol. 1 no. 2, pp. 205-214.

[6] C. F. Eick, N. Zeidat, Z. Zhao, "Supervised Clustering-Algorithms and Benefits", in Proceedings of the IEEE International Conference on Tools with Artificial Intelligence, Boca Raton, Florida, USA, pp. 774 776, November 2004

[7] J. Fridrich, "Digital Image Forensics", IEEE Signal Processing Magazine, IEEE, 2009, vol. 26, no. 2, pp. 26-37.

[8] C.-T. Li, "Unsupervised Classification of Digital Images Using Enhanced Sensor Pattern Noise", in Proceedings of the IEEE International Symposium on Circuits and Systems, Paris, France, May 2010, pp. 3429-3432.

[9] L. Rokach, "A Survey of Clustering Algorithms", Data Mining and Knowledge Discovery Handbook, 2010, pp. 269-298.

[10] C. T. Zahn, "Graph-Theoretical Methods for Detecting and Describing Gestalt Clusters", IEEE Transactions on Computers, IEEE, 1971, vol. C-20, no. 1 , pp. $68-86$. 
[11] J. D. Banfield, A. E. Raftery, "Model-Based Gaussian and NonGaussian Clustering", Biometrics, Wiley, 1993, vol. 49, no. 3, pp. 803821.

[12] D. H. Fisher, "Knowledge Acquisition Via Incremental Conceptual Clustering", Machine Learning, Springer, vol. 2. no. 2, pp. 139-172, 1987.

[13] J. Vesanto, E. Alhoniemi, "Clustering of the Self-Organizing Map", IEEE Transactions on Neural Networks, IEEE, 2000, vol. 11, no. 3, pp. 586-600.

[14] F. Hoppner, "Fuzzy Cluster Analysis: Methods for Classification", Data Analysis and Image Recognition, Jossey-Bass Higher and Adult Education Series, Wiley, 1999.
[15] S. Z. Selim, K. Alsultan, "A Simulated Annealing Algorithm for the Clustering Problem", Pattern Recognition, Elsevier, 1991, vol. 24, no. 10, pp. 1003-1008.

[16] B.-B. Liu, H.-K. Lee, Y. Hu, C.-H. Choi, "On Classification of Source Cameras: A Graph Based Approach", in Proceedings of the IEEE International Workshop on Information Forensics and Security, Seattle, Washington, USA, December 2010, pp. 1-5.

[17] R. Caldelli, I. Amerini, F. Picchioni, M. Innocenti, "Fast Image Clustering of Unknown Source Images", in Proceedings of the IEEE International Workshop on Information Forensics and Security, Seattle, Washington, December 2010, USA, pp. 1-5.

[18] M. Goljan, J. Fridrich, T. Filler, "Large Scale Test of Sensor Fingerprint Camera Identification", in Proceedings of the Media Forensics and Security, vol. 7254, San Jose, California, USA, 2009, pp. 72540I. 